\title{
INCIDENCIA EN INFECCIONES RESPIRATORIAS AGUDAS Y PRESCRIPCIÓN DE MEDICAMENTOS EN NIÑOS MENORES DE CINCO AÑOS QUE ACUDAN AL PUESTO DE SALUD LAS BEGONIAS, NOVIEMBRE 2012 - DICIEMBRE 2013
}

\author{
INCIDENCE IN ACUTE RESPIRATORY INFECTIONS AND PRESCRIPTION \\ OF MEDICINES IN CHILDREN UNDER FIVE YEARS OLD WHO COME TO \\ LAS BEGONIAS HEALTH POST. NOVEMBER, 2012 - DECEMBER, 2013.
}

\author{
${ }^{1}$ Edgard Guido Calderón Copa; ${ }^{2}$ Juan José Evaristo Changllio Roas; ${ }^{3}$ Pablo Stiwar Vicente Calderón;
}

${ }^{4}$ Gina Marisol Chambilla Ticona

\begin{abstract}
RESUMEN
El presente trabajo de investigación es un estudio básico, de carácter descriptivo no experimental y prospectivo que se efectuó de noviembre 2012 a diciembre del 2013, en el Puesto de salud "Las Begonias", del Distrito Gregorio Albarracín Lanchipa de la Provincia y Región Tacna. La muestra fue de 350 niños menores a cinco años con IRA, cuya población total fue de 1371. Para determinar la frecuencia en la incidencia de IRAs en niños menores de cinco años de edad se utilizó como instrumento las historias clínicas de la cual se obtuvo la información necesaria mediante fichas de recolección, luego se tabularon, analizaron e interpretaron de acuerdo a los objetivos planteados. Se obtuvieron los siguientes resultados: 350 niños menores de cinco años presentaron IRAs, cuyo porcentaje fue $25,52 \%$, valor mayor al planteado en la hipótesis; $10,00 \%$. La frecuencia de IRAs en los niños de 0 a 1 año fue $33,14 \%$; de 1 a 2 años, $22,00 \%$. La faringoamigdalitis es la IRA más frecuente (37,98 \%), luego faringitis aguda $(19,40 \%)$. El medicamento más prescrito fue ibuprofeno jarabe $(37,50 \%)$. El número de fármacos prescritos fueron: dos $33,14 \%$; tres, $22,00 \%$. Finalmente referente las patologias que se presentaron paralelamente con la IRAS fueron: anemia por deficiencia de hierro $45,57 \%$; parasitosis intestinal $17,72 \%$ y caries dental $11,39 \%$.
\end{abstract}

Palabras clave: Infecciones respiratorias agudas, prescripción de fármacos.

\begin{abstract}
The present Research is a typical basic study of descriptive, non experimental and prospective character that was carried out during November 2012 to December 2013, in las Begonias health post of Gregorio Albarracin Lanchipa district of the Province and Tacna's Department. The sample consisted of 350 children under five years old with acute respiratory infections (ARIS) whose total population was of 1371. To determine the frequency of ARIS incidence in children under five years old, the medical records were used as instruments from which the necessary information was obtained by means of records of compilation, and then they were tabulated, analyzed and interpreted according to the objectives. The following results were obtained; 350 children under five-year-olds presented ARIS, whose percentage was $25,52 \%$, a value bigger than the raised one in the hypothesis; $10,00 \%$. The frequency of ARIS in children from 0 to 1 years old was $33,14 \%$, from I to 2 years; $22,00 \%$. The Faringoamigdalitis is the most frequent ARI 37,98 \%, then acute Pharyngitis $19,40 \%$. The most prescribed medicine was ibuprofen syrup $37,50 \%$. The number of prescribed medicaments were; two $33,14 \%$, three, $22,00 \%$. Finally about the pathologies that were presented in parallel withARIS; iron deficiency anemia $45,57 \%$, intestinal parasitosis $17,72 \%$ and dental caries $11,39 \%$.
\end{abstract}

Key words: acute respiratory infections (ARIS), prescription of medicaments.

\section{INTRODUCCIÓN}

El epitelio del tractus respiratorio constituye la mayor superficie anatómica del organismo expuesta a los agentes externos y no es sorprendente, que las infecciones respiratorias agudas (IRAs) sean las más comunes que afectan al ser humano.

El aparato respiratorio está expuesto a infinidad de agentes infecciosos que cuando logran superar los mecanismos defensivos de este, se producen diversas enfermedades causadas por infinidad de microorganismos entre ellos virales y bacterianos. (17)

Las IRAs son un complejo y heterogéneo grupo de enfermedades causadas por distintos gérmenes, que afectan cualquier parte del aparato respiratorio y se describen entidades de acuerdo con el lugar donde predominan los

'Quimico Farmacéutico. Facultad de Ciencias de la Salud de la Universidad Nacional Jorge Basadre Grohmann. Tacna-Perú.

${ }^{2}$ Magister Scientiae en Tecnologia Educativa, Segunda Especialidad en Toxicología y Control Alimentario. Facultad de Ciencias de la Salud de la Universidad Nacional Jorge Basadre Grohmann. Tacna-Perú.

${ }^{3}$ Biỏlogo Microbiólogo. Facultad de Ciencias de la Salud y Medicina de la Universidad Alas Peruanas, Filial Tacna-Perú.

${ }^{4}$ Obstetriz. Ministerio de Salud. Tacna-Perú 
Calderón, E. et al., Incidencia en infecciones respiratorias agudas y prescripción de medicamentos en niños menores de 5 años que acudan al P. S. Las Begonias, Nov.2012-Dic 2013.

síntomas. (5)

Desde el resfriado común hasta la influenza, la infección respiratoria es una experiencia universal y en la mayoría de los casos una enfermedad menor, aunque a veces molesta, auto limitada casi siempre. (5)

En una época donde las enfermedades emergentes y reemergentes reciben la mayor atención de la comunidad cientifica, por causa de la enorme trascendencia social en el mundo de hoy y su repercusión futura, las IRAs se mantienen como un grupo importante de afecciones con una alta morbilidad y baja mortalidad, las que representan un motivo frecuente de incapacidad laboral y escolar con las consecuentes pérdidas económicas que ello significa. Estas afecciones, conjuntamente con las enfermedades diarreicas y la malnutrición, encabezan las principales causas de muerte entre los niños en los países subdesarrollados.

A medida que aumenta el número de factores de riesgo presentes en un niño se incrementa el riesgo de morbilidad grave y muerte, por lo que el médico, siempre debe de valorar la presencia de los mismos en todo paciente que acude a consulta con un proceso infeccioso respiratorio agudo. (14)

Constituyen la primera causa de consultas médicas y de morbilidad tanto en países desarrollados como en los países en vías de desarrollo. La presencia de estas en menores de cinco años es independiente de las condiciones de vida y grado de desarrollo. La diferencia no radica en el número de episodios sino en su gravedad. (5)

Existen 4 aspectos importantes en el análisis de las IRAs, y a la vez en el enfoque de las estrategias de prevención y control: la presencia de factores de riesgo, la morbilidad, la mortalidad y la calidad de la atención médica.

Las IRAs se clasifican en altas y bajas según afecten fundamentalmente, en el sistema respiratorio, estructuras por encima de la laringe o por debajo de esta. A su vez estas se dividen en no complicadas y complicadas. (14)

El mecanismo de transmisión es fundamentalmente por contacto directo, el período de incubación es variable y puede durar hasta 14 días. Varios son los factores de riesgo en la IRA como son: demográficos, socioeconómico (ingreso familiar bajo, nivel de escolaridad, lugar de residencia), ambientales que incluye exposición al humo (contaminación atmosférica, contaminación doméstica por residuos orgánicos, humo ambiental por tabaco), hacinamiento (aglomeración de personas), exposición al frio, humedad y cambios bruscos de temperatura, deficiente ventilación en la vivienda y factores nutricionales. (12)

Constituyen un problema de prioridad a nivel mundial, incidiendo en la morbimortalidad infantil y representan el $40-60 \%$ de las consultas, la incidencia es más elevada en las áreas urbanas, el número de episodios puede ser entre 2 y 6 crisis anuales. (9)

Existen factores de riesgo que predisponen o favorecen las Infecciones, algunos modificables y otros no, como son: edad, bajo peso al nacer, desnutrición, déficit inmunológico, hacinamiento, contaminación ambiental, uso de keroseno, humo del cigarro o tabaco, (ya sea de forma pasiva o activa) así como la presencia de alguna enfermedad de base (8). A nivel mundial, se considera que más del $90 \%$ de los casos de niños menores de 5 años que acuden a consulta es por IRAs. (15)

La etiología viral es la más frecuente, particularmente las del aparato respiratorio superior. (16)

Los gérmenes más frecuentes en el aparato respiratorio son: virus Sincitial Respiratorio, específicamente en el niño menor de un año, Parainfluenza, Adenovirus, Rinovirus, Coronavirus, Enterovirus. (11)

En las infecciones del aparato respiratorio inferior, los virus también son los más frecuentes en la mayoría de los casos, sin embargo, se encuentra un porcentaje elevado de etiología bacteriana, lo cual justifica un tratamiento diferente (10). La infección se adquiere probablemente por la inhalación de secreciones nasofaríngeas infectadas, dado que las secreciones de las vías respiratorias superiores pueden contener grandes cantidades de patógenos (1). La aspiración de una pequeña cantidad podría ser un inoculo suficiente para causar la enfermedad, especialmente si las defensas locales del tracto respiratorio inferior están afectadas por desnutrición, procesos virales y otros factores. (18)

Dentro de los agentes bacterianos en las infecciones adquiridas en la comunidad los microorganismos más comunes son Streptococcus Pneumoniae, Haemopbilus Influenzae y Staphylococcus Aureus. Otros agentes causales son Micoplasma Pneumoniae, Clamidias, Rickettsias y hongos. (14)

Existe un gran esfuerzo para disminuir los factores de riesgo de esta entidad, para así reducir el ingreso hospitalario y mejorar su diagnóstico y tratamiento (7). Debido a todo esto es importantísimo el conocimiento de los factores de riesgo y la influencia de ellos en la aparición y exacerbación de las IRAs, sobre todo en niños lactantes y en menores de cinco años. (8)

Las IRAS son un grupo de enfermedades del aparato respiratorio causadas por microorganismos virales o bacterianos, los cuales se incrementan en la temporada de frío, además de la limitada cultura preventiva, frente a la presencia de cambios bruscos de temperatura en el Perú, lo cual es recurrente en los últimos años. Son las primeras causas de muerte en todas las etapas de vida y su mayor incidencia y letalidad se presenta en los menores de 05 años y adultos mayores, a consecuencia de la diversidad meteorológica; como la temporada de frío, heladas, granizadas, nevadas y friaje en las regiones de la sierra, y selva.

A nivel nacional (Perú) en las dos primeras semanas del mes de enero del año 2011, se notificaron 111357 atenciones por IRAs en menores de 5 años, que representan una tasa de 378 atenciones por IRA por cada 10000 menores de 5 años.

El mayor número de estas atenciones (110 261), fueron por IRAs no complicadas (incluyen infecciones respiratorias agudas de vías respiratorias altas: resfrío común, faringitis aguda, bronquitis aguda y otitis media).

Los episodios de neumonías notificados hasta la semana 2 fueron 1096, y representan una tasa de casi 4 episodios por cada 10000 menores de 5 años.

Los departamentos con las más altas tasas de neumonía son: Pasco, Ucayali, Callao y Loreto, reportando casi 3 veces el promedio nacional (entre 8 a 10 atenciones por cada 10000 menores de 5 años). 
Otra afección respiratoria sujeta a vigilancia epidemiológica es el síndrome obstructivo bronquial y el asma. El total de atenciones en menores de cinco años notificadas hasta la segunda semana del año 2011 fue de 7982 , que representa una tasa de 27 atenciones por cada 10000 menores de cinco años.

Cada niño padece unas 6 a 8 IRAs anualmente hasta los cinco años. Este grupo de enfermedades supone $30-50 \%$ de las consultas pediátricas en los países pobres y $20-40 \%$ de los ingresos hospitalarios en pediatría. (4)

En el puesto de salud "Las Begonias" del distrito Gregorio Albarracín Lanchipa, se ha reportado como primera causa de morbilidad y mortalidad a las IRAS en niños menores de cinco años, hecho que nos llevó a estudiar y evidenciar su frecuencia, así como determinar que enfermedad es la mayor causante de estas infecciones, además de identificar cuáles son los medicamentos más prescritos para estas patologías.

\section{MATERIAL Y MÉTODOS}

El presente trabajo fue enfocado a niños menores de cinco años que acudieron al puesto de salud Las Begonias, de noviembre 2012 a diciembre del 2013. La población fue de 1371 niños menores de cinco años, de ello se tomó una muestra de 350 niños que han sufrido por lo menos un episodio de IRA. Se tomaron los datos de sus historia clínicas, de acuerdo a los objetivos planteados, en fichas de información que fueron posteriormente analizados. Los resultados obtenidos se expresan estadísticamente utilizando la media aritmética, tablas de frecuencia relativas y acumuladas.

\section{RESULTADOS}

Tabla N ${ }^{\circ} 01$. Frecuencia de IRAs en menores de cinco años.

\begin{tabular}{lcc}
\hline $\begin{array}{l}\text { Niños menores de cinco } \\
\text { años con o sin ITUS }\end{array}$ & $\begin{array}{c}\text { Niños menores } \\
\text { de cinco años }\end{array}$ & $\%$ \\
\hline Con IRAS & 350 & 25,52 \\
Sin IRAS & 1021 & 74,48 \\
Total & 1371 & 100,00 \\
\hline
\end{tabular}

En la tabla $\mathrm{N}^{\circ} 01$, se aprecia el número de niños menores de cinco años totales (1371), que acudieron al Puesto de Salud "Las Begonias", de las cuales 350 presentaron IRAs, el cual representa $25,52 \%$. Debemos mencionar que 1021 que representan el $74,48 \%$ de niños menores de cinco años, no presentaron IRAs.

En la tabla $\mathrm{N}^{\circ} 02$, se aprecia que la totalidad de niños menores de cinco años con IRAs que recibieron medicamentos como tratamiento para tal patología, fue el $25,52 \%$ (350) del total de la población infantil en estudio. Lo que también se evidencia, es que 1021 casos, que representan el $74,48 \%$, no recibieron medicación alguna.

La tabla $\mathrm{N}^{\circ} 03$ evidencia la incidencia de las IRAs de acuerdo al grupo etario, y se encontró que el grupo etario de 0 a 1 años presentó una mayor frecuencia, $33,14 \%$, seguido del grupo de 1 a 2 años con un $22,00 \%$, y si comparamos con la población total de menores de cinco
Tabla $\mathbf{N}^{\circ} \mathbf{0 2}$. Frecuencia en la prescripción de fármacos

\begin{tabular}{lcc}
\hline $\begin{array}{l}\text { Prescripción de } \\
\text { fármacos }\end{array}$ & $\begin{array}{c}\text { Niños menores de } \\
\text { cinco años }\end{array}$ & $\%$ \\
\hline Sin prescripción & 1021 & 74,48 \\
Con prescripción & 350 & 25,52 \\
Total & 1371 & 100,00 \\
\hline
\end{tabular}

Tabla $\mathbf{N}^{\circ} 03$. Frecuencia de IRAS de acuerdo al grupo etario

\begin{tabular}{lccl}
\hline \multicolumn{1}{c}{ Grupo etario } & Con IRAS & $\%$ & Al $\mathbf{1 0 0} \%$ \\
\hline 0 a 1 años & 116 & 33,14 & 8,46 \\
1 a 2 años & 77 & 22,00 & 5,62 \\
2 a 3 años & 68 & 19,43 & 4,96 \\
3 a 4 años & 45 & 12,86 & 3,28 \\
4 a 5 años & 44 & 12,57 & 3,21 \\
Total & 350 & 100,00 & 25,53 \\
\hline
\end{tabular}

Tabla $\mathrm{N}^{\circ} 04$. Frecuencia de tipos de IRAS

\begin{tabular}{lcc}
\hline Tipo de IRAS & Con IRAS & $\%$ \\
\hline Faringoamigdalitis & 147 & 37,98 \\
Faringitis aguda & 75 & 19,40 \\
Rinofaringitis aguda & 70 & 18,09 \\
Amigdalitis aguda & 38 & 9,82 \\
Bronquitis aguda & 22 & 5,68 \\
Rinitis alérgica & 17 & 4,39 \\
Resfrió común & 6 & 1,55 \\
Asma bronquial & 6 & 1,55 \\
Traqueitis aguda & 4 & 1,03 \\
Laringotraqueitis aguda & 2 & 0,51 \\
Total & 387 & 100,00 \\
\hline
\end{tabular}

años, podemos mencionar que el grupo etario de 0 a 1 año $y$ de 1 a 2 años, son los que representan mayores porcentajes de incidencias de IRAs, 8,46 y 5,62 respectivamente.

En la tabla $\mathrm{N}^{\circ} 04$ se muestra, la frecuencia de tipos de IRAs, y se encuentra que la faringoamigdalitis es la más frecuente, $37,98 \%$ (147), seguido de la faringitis aguda 19,40 $\%(75)$, rinofaringitis aguda y bronquitis aguda con $18,09 \%$ y $9,82 \%$ respectivamente.

En la tabla $\mathrm{N}^{\circ} 05$ se muestra que el ibuprofeno en jarabe es el medicamento más prescrito $25,37 \%$, seguido por la amoxicilina de $250 \mathrm{mg}$ en suspensión, clorfenamina y paracetamol jarabe con $19,53 \%, 13,45 \%$ y $11,90 \%$, respectivamente. Casi la totalidad de medicamentos están relacionados con IRAs; pero, albendazol en suspensión, clotrimazol en crema y sulfato ferroso en jarabe con $0,36 \%$ cada uno de ellos indican la presencia de otras patologías.

En la tabla $\mathrm{N}^{\circ} 06$, se muestra los resultados obtenidos en referencia al número de fármacos prescritos, encontrándose que es más frecuente la prescripción de dos fármacos con $64,17 \%$, seguido por la de tres fármacos $22,68 \%$.

En la tabla $\mathrm{N}^{\circ} 07$, se muestra a las otras patologias más frecuentes en los niños menores de cinco años que tienen paralelamente a las IRAs, y se evidencia que se ha encontrado $36 \operatorname{casos}(45,57 \%)$, que han sido prescritas con sulfato ferroso en suspensión, seguida por la parasitosis intestinal $(17,72 \%)$. 
Tabla N 5 . Fármacos más prescritos

\begin{tabular}{lcc}
\hline Fármaco & CASOS & $\%$ \\
\hline Ibuprofeno jarabe & 213 & 25,37 \\
Amoxicilina 250mg jarabe & 164 & 19,53 \\
Clorfenamina jarabe & 113 & 13,45 \\
Paracetamol jarabe & 100 & 11,90 \\
Eritromicina 250mg jarabe & 65 & 7,74 \\
SMT/TMT 200/40 suspensión & 42 & 5,00 \\
Dextrometorfano jarabe & 24 & 2,86 \\
Dexametasona ampolla & 22 & 2,62 \\
Paracetamol gotas & 20 & 2,38 \\
P. Procainica 1000000ui Amp. & 18 & 2,14 \\
Ambroxol 15mg jarabe & 10 & 1,19 \\
Azitromicina jarabe & 6 & 0,71 \\
Fluimucil 100 Mg sobres & 6 & 0,71 \\
Salbutamol Inhalador & 6 & 0,71 \\
Loratadina jarabe & 6 & 0,71 \\
Bacterol Balsamico jarabe & 6 & 0,71 \\
Cetirizina Jarabe & 6 & 0,71 \\
Beclometasona inhalador & 4 & 0,48 \\
Albendazol suspension & 3 & 0,36 \\
Clotrimazol crema & 3 & 0,36 \\
Sulfato Ferroso jarabe & 3 & 0,36 \\
Total & 840 & 100,00 \\
\hline
\end{tabular}

\section{DISCUSIÓN}

Las infecciones respiratorias agudas (IRA) representan uno de los principales problemas de salud entre los niños menores de 5 años de los países en desarrollo. En la Región de las Américas, las IRAs se ubican entre las primeras cinco causas de defunción de menores de 5 años y representan la causa principal de enfermedad y consulta a los servicios de salud (2).

La neumonía es la principal causa de muerte en los niños de todo el mundo y la gran mayoría de estas muertes ocurren en países en desarrollo. Se estima que cada año fallecen por esta causa 1,4 millones de niños menores de cinco años, más que las causadas por el VIH/SIDA, malaria y sarampión juntos, lo que supone el $18 \%$ de todas las defunciones de niños menores de cinco años en todo el mundo (23). Para el año 2010, a nivel global se estimó 1071 millones de defunciones por neumonía en menores de 5 años (rango de incertidumbre de 977-1176 millones) (13).

Aunque se han hecho estimados globales sobre las defunciones por neumonía en los primeros 5 años de su vida (3). La verdadera mortalidad causada por neumonía, probablemente está subestimada, ya que en los países en desarrollo la mayoría de las muertes se presentan en el hogar, sin un diagnóstico médico. El $90 \%$ de las muertes se cree que ocurre en el mundo en desarrollo y el $50 \%$ de ellas se producen en África. (2)

En América Latina fallecen 72000 niños anualmente por causa de neumonía, principalmente de etiología
Tabla $\mathbf{N}^{\circ} 06$. Número de fármacos prescritos

\begin{tabular}{ccl}
\hline Número de Fármacos & Gestantes & $\%$ \\
\hline 0 & 2 & 0,52 \\
1 & 24 & 6,19 \\
2 & 249 & 64,17 \\
3 & 88 & 22,68 \\
4 & 21 & 5,41 \\
5 & 4 & 1,03 \\
Total & 388 & 100,00 \\
\hline
\end{tabular}

Tabla $N^{\circ} 07$. Otras enfermedades paralelas a las IRAS

\begin{tabular}{lcl}
\hline Otras Enfermedades & Gestantes & $\%$ \\
\hline Anemia por deficiencia de Hierro & 36 & 45,57 \\
Parasitosis Intestinal & 14 & 17,72 \\
Caries Dental & 9 & 11,39 \\
Diarrea Acuosa S/DH & 6 & 7,59 \\
Estreñimiento & 5 & 6,33 \\
Dermatitis Alérgica & 4 & 5,06 \\
Micosis Superficial & 2 & 2,53 \\
Micosis Cutánea & 1 & 1,27 \\
Conjuntivitis & 1 & 1,27 \\
Aftas & 1 & 1,27 \\
Total & 79 & 100,00 \\
\hline
\end{tabular}

bacteriana. La Organización Panamericana de la Salud (OPS) estima que el Streptococcus pneumoniae causa alrededor de 20000 muertes anuales. $(20,21)$

En nuestro país, la prevención y control de las IRA en menores de 5 años es una prioridad, no sólo porque son la primera causa de morbi - mortalidad, sino por la alta demanda y el uso frecuente de los servicios de salud.

En la tabla $\mathrm{N}^{\circ} 01$, se observa un resultado muy importante con relación a la Incidencia en la Frecuencia de las IRAs en niños menores de cinco años que acudieron al P. de S. "Las Begonias", cuyo resultado es que de 1371 niños, 350 resultaron con IRAs el cual es un $25,52 \%$, valor muy elevado. Hecho que ratifica los numerosos estudios que reportan la asociación entre variaciones climáticas (temperatura del aire y humedad) y morbimortalidad por enfermedades respiratorias, cardiovasculares y cerebrovasculares, así como la asociación entre contaminación ambiental e incremento de la incidencia de asma y enfermedades alérgicas, siendo los extremos de la vida (niños y adultos mayores) los más afectados. Sin embrago, los resultados no son uniformes, debido probablemente a la interacción de otros factores como las características socioeconómicas y culturales de las poblaciones estudiadas, que tendrían un impacto en las asociaciones mencionadas. (6)

Con relación a la frecuencia relacionada a la prescripción de medicamentos a niños menores de cinco años con IRAs (tabla $\mathrm{N}^{\circ} 02$ ), se evidencia que el $25,52 \%$ de niños con IRAs fueron prescritos, valor muy significativo respecto a la totalidad de niños (1371) y ello debido, entre muchos factores a la mala alimentación y factores higiénicos, 
falta de lactancia materna, medio ambiente externo afectado por la contaminación o epidemias, y el medio ambiente interno en el hogar. Es muy importante mencionar que este valor encontrado es mayor al valor propuesto, con más de $10,00 \%$.

En la tabla $\mathrm{N}^{\circ} 03$, se puede observar que el $33,14 \%$ de niños menores de cinco años con IRAs pertenecen al grupo etario de 0 a 1 año, es decir, que en este grupo etario hay mayor predominio de IRAs, también es importante mencionar que el porcentaje encontrado en el grupo etario de 1 a 2 años es de $22,00 \%$, y ello es debido a la corta edad, la mayoría de las IRAs ocurren en el primer año de vida y, sobre todo, en los primeros 6 meses, debido a la inmadurez de las vías respiratorias y sus mecanismos defensivos, lo que favorece la mayor gravedad y la predisposición a estas infecciones. Estos resultados están en correspondencia con lo planteado en la literatura mundial donde se plantea que en los primeros años de vida el niño tiene menos madurez inmunológica, es decir se defiende menos con relación a las enfermedades infecto contagiosas y son similares a lo reportado en estudios realizados por la Organización Panamericana de la Salud (OPS) en Bolivia donde la mayor incidencia fue en los niños menores de 2 años con un $76 \%$ de la muestra escogida por el autor Benguigui. (3)

Otro estudio similar hecho en Chile por Ruth Campillo Moliera (22) en el año 2003, encontró que la prevalencia por edad, en el grupo de 0 a 2 años con una muestra de 218 niños, fue de $77,3 \%$ del universo.

La tabla $\mathrm{N}^{\circ} 04$, evidencia la frecuencia en el tipo de IRA, en el que la faringoamigdalitis y la faringitis aguda con $37,98 \%$ y $19,40 \%$ respectivamente, se presentan con mayor frecuencia, debido a la predisposición fisiológica natural de los niños menores de cinco años. Una investigación realizada por Gloria Robaina en el Policlínico Docente Mario Escalona Reguera en el año 2001, encontró que el catarro común y la rinofaringitis aguda prevalecieron con un $66 \%$ del total de las IRAs (19), al igual que en Candelaria y otros países de América y el Caribe donde las IRAs altas siguen predominando en niños menores de cinco años de edad, encabezadas por las infecciones virales y entre ellas la rinofaringitis aguda de primer orden.

La tabla $\mathrm{N}^{\circ} 05$, sobre los fármacos más prescritos en niños menores de cinco años con IRAs, muestra que los medicamentos más prescritos son el ibuprofeno jarabe, amoxicilina $250 \mathrm{mg}$ jarabe, clorfenamina jarabe $y$ paracetamol jarabe con $25,37 \%, 19,53 \%, 13,45 \%$ y $11,90 \%$ respectivamente. Ello hace notar que se siguen los protocolos del Ministerio de Salud, y se hacen recomendaciones como reposo y aislamiento, dieta y líquidos, antitérmicos, control de la tos (excepcional), educación, control de la ansiedad e intercambio de opiniones con los familiares. Dependiendo de la fiebre se han prescrito amoxicilina $250 \mathrm{mg}$ jarabe con paracetamolo ibuprofeno

En el tabla $\mathrm{N}^{\circ} 06$, se observa la prescripción de fármacos en función de su cantidad prescrita, y se encontró que se prescriben dos o tres medicamentos en mayor frecuencia con un $64,17 \%$ y $22,68 \%$ respectivamente y las polifarmacias son amoxicilina $250 \mathrm{mg}$ jarabe con paracetamol o ibuprofeno jarabe y clorfenamina jarabe, y la otra opción ibuprofeno jarabe o paracetamol jarabe con clorfenamina jarabe, dependiendo del tipo de IRA. Obviamente ello está en función de la patología para llegar a un buen tratamiento que erradique la infección.

Finalmente la tabla $\mathrm{N}^{\circ} 07$, reporta información importante referida a la presentación paralela de otras patologías en los niños menores de cinco años con las IRAs, podemos mencionar que es característico la presencia de anemia por deficiencia de hierro, parasitosis intestinal, caries dental y diarrea acuosa preponderantemente con valores de $45,45 \%, 17,72 \%, 11,39 \%$ y $7,59 \%$ respectivamente; patologías características en Tacna especialmente en los conos, ya sea Norte y Sur, debido a la falta de una buena nutrición suplementada con vitaminas y minerales, también a la falta de una buena higiene personal, falta de agua, falta de instalaciones de alcantarillado.

\section{CONCLUSIONES}

Referente a la frecuencia de IRAs en niños menores de cinco años en el puesto de salud "Las Begonias", el $25,52 \%$ de niños menores de cinco años presentaron IRAs y el $74,48 \%$ no.

Referente a la frecuencia en la prescripción de fármacos en el puesto de salud "Las Begonias", el 25,52\% representan a niños menores de cinco años que presentan IRAs y que fueron prescritas en el mismo porcentaje.

En referencia a la frecuencia de IRAs de acuerdo al grupo etario, los niños que están en el grupo de 0 a 1 año, representan el 33,14\% y los niños menores de cinco años que pertenecen al grupo etario de 1 a 2 años el $22,00 \%$.

Con referencia a la frecuencia de IRAs de acuerdo a sus diferentes tipos, se encontró que la faringoamigdalitis es la patología que más frecuentemente se presenta, con un $37,98 \%$; la faringitis aguda representa el $19,40 \%$ y la rinofaringitis aguda el $18,09 \%$.

Referente al medicamento más prescrito, al $37,50 \%$ de niños menores de cinco años con IRA, les prescribieron ibuprofeno jarabe; al 19,53\% les prescribieron amoxicilina $250 \mathrm{mg}$ suspensión, y al $13,45 \%$, les prescribieron clorfenamina jarabe.

Referente al número de medicamentos prescritos, la prescripción de dos fármacos es la más frecuente, $64,17 \%$, seguida de tres fármacos, $22,68 \%$.

En referencia a otras patologias presentes paralelamente a las IRAs, la anemia por deficiencias de hierro, representa el $45,57 \%$; la parasitosis intestinal, el $17,72 \%$; la caries, $11,39 \%$. Y las de menor frecuencia son: diarrea acuosa, estreñimiento, dermatitis alérgica y micosis superficial, con $7,59 \%, 6,33 \%, 5,06 \%$ y $2,53 \%$ respectivamente.

\section{RECOMENDACIONES}

- Se recomienda difundir en los padres de familia la importancia sobre la predisposición que hay en los niños menores de cinco años a adquirir fácilmente cualquier tipo de IRA si las condiciones les permiten.

- Cuestionar y evitar que los padres de familia automediquen a los niños, el cual puede conllevar al uso 
Calderón, E. et al., Incidencia en infecciones respiratorias agudas y prescripción de medicamentos en niños menores de 5 años que acudan al P. S. Las Begonias, Nov.2012-Dic 2013.

irracional de antibióticos y demás fármacos, con presencia de RAMS.

- Seguida a la prescripción, orientar detenidamente sobre su dosificación, la hora exacta en la que debe tomar el medicamento, las contraindicaciones que pudiese tener e interacciones.

- Realizar trabajos de investigación sobre "fármacovigilancia", para lograr un uso adecuado de los medicamentos, dándole énfasis al uso de los mismos en niños menores de cinco años.

- Ampliar la investigación sobre IRAs en niños menores de cinco años, a otros Centros de Salud de la Región Tacna.

\section{REFERENCIAS BIBLIOGRÁFICAS}

1. Arnold JE. Infections of the upper respiratory tract. En: Nelson W. Textbook of Pediatrics. 15th edition, W.B. Saunders Company 2000; 1187-93.

2. Benguigui Y, López Antuñano FJ, Schmunis G, Yunes J. Infecciones respiratorias en niños. Washington, D.G. OPS. 1997.

3. Benguigui Y. Infecciones respiratorias agudas. En: Meneghedo J, et al. Diálogo en Pediatría. Santiago de Chile. Publicaciones técnicas mediterráneas. 1998; (3): 11-26.

4. Boletín Epidemiológico. Dirección General de Epidemiología, Red Nacional de Epidemiología, Ministerio de Salud. Lima. 2011;20 (2):23-24.

5. Correa AG, Starke JR. Bacterial pneumonias. In Kendig's Disorders of the Respiratory Tract in Children. 6th edition. Saunders Company. 2002; Pags 485-503.

6. Delgado HL, Girón EM, Ramirez HL, Hurtado E. Epidemiology of acute respiratory infections in preschool children of rural Guatemala. PAHO Bulletin 1988;22:383-93.

7. Derdy, Robert. El Manual Merck. Ed. Interamericana. $7^{\circ}$ Edición. 2002.

8. Diccionario de Medicina Mosby. Edición 2005.

9. Drummond P, Clark J, Wheelar J, Galloway A, Freeman $\mathrm{R}$, Cant A. Community acquired pneumonia- a prospective UK study. Arch Dis Child 2005; 83: 408-12.

10. Fernández, Alfonso. Infecciones respiratorias agudas. Gripe, resfrío e influenza. Gripe. Procedimientos y medicamentos Manual de Diagnóstico y Tratamiento en
Especialidades Clínicas; 2005; 107-36.

11. Fernández, Alfonso. Manual de Diagnóstico y Tratamiento en Especialidades Clínicas. 2005; 56-68.

12. Lerou PH. Lower respiratory tract infections in children. Curr Opin Pediatr. 2004; 13:200-6.

13. Liu L, Johnson HL, Cousens S, Perin J, Scott S, Lawn JE, et al. Global, regional, and national causes of child mortality: an updated systematic analysis for 2010 with time trends since 2000. The Lancet. 2012.

14. Mc Intosh K. Community-acquired pneumonia in children. N Engl J Med. 2002;346:429-37.

15. Ministerio de Salud. República del Perú. Doctrina, Normas y Procedimientos para el Control de la Infección Respiratoria Aguda, Síndrome Obstructivo Bronquial y Asma; en menores de 5 años. Lima - Perú. 2000.

16. Ministerio de Salud. República del Perú. Manual de SUBPCIRA. 2000.

17. Palomino MA, Larrañaga C. Infección intra y extrahospitalaria por VRS en lactantes. Rev Chil Pediatr. 2004; 65:11-16.

18. Pickering LK, Morrow AL. Child care and communicable diseases. En: Nelson W. Textbook of Pediatrics. 15th edition, W.B. Saunders Company. 2000; 1028-30.

19. Rosenfeld JA, Clarity G. Acute otitis media in children. Prim care. 2001;23(4): 677-86.

20. Rudan 1, Boschi-Pinto C, Biloglav Z, Mulholland K, Campbell H. Epidemiology and etiology of childhood pneumonia . Bulletin of the World Health Organization. [Research Support, Non-U.S. Gov't]. 2008 May; 86(5):408-16.

21. Saha SK, Naheed A, El Arifeen S, Islam M, Al-Emran H, Amin R, et al. Surveillance for invasive Streptococus pneumoniae disease among hospitalized children in Bangladesh: antimicrobial susceptibility and serotype distribution. Clinical infectious diseases: an official publikation of the lnfectious Diseases Society of America. 2009 Mar 1; 48 Suppl 2:S75-81.

22. Suárez Glañaria R; Campillo Moliera R; Vejar M.; Las enfermedades respiratorias agudas de la infancia. Santiago de Chile: Pan Am J Public Health 2001; 3(2).

23. Wardlaw T, Salama P, Johansson EW, Masan E. Pneumonia: the leading killer of children. Lancet. 2006 Sep 23; 368 (9541):1048-50.

\section{Correspondencia:}

Edgard Guido Calderón Copa: edgardguidocald@hotmail.com
Fecha de Recepción: 21/07/2014

Fecha de Aceptación: 21/11/2014 